\title{
An Introduction to the Source Concept for Antennas
}

\author{
Pavel HAZDRA, Miloslav CAPEK, Michal MASEK, Tomas LONSKY
}

Dept. of Electromagnetic Field, Czech Technical University in Prague, Technicka 2, 16627 Prague, Czech Republic

hazdrap@fel.cvut.cz

Manuscript received March 4, 2016

\begin{abstract}
Antenna parameters particularly relevant to electrically small antenna design are reviewed in this paper. Source current definitions are accentuated leading to the introduction of the source concept which advantageously utilize only spatially bounded quantities. The framework of the source concept incorporates powerful techniques such as structural and modal decomposition, operator's inversion and current optimization, thus opening new, challenging possibilities for antenna design, analysis and synthesis.
\end{abstract}

\section{Keywords}

Poynting theorem, antenna theory, stored energy, quality factor $Q$, source concept

\section{Introduction}

Certain antenna parameters, including for example radiated power, are traditionally calculated from radiated fields in space. Recently, a number of papers, see e. g. [1], [2] have been published which attempt to use only antenna geometry and source distribution (electric or magnetic or both currents) when evaluating the performance of antennas using spatially limited integrations over current density. This is advantageous as you do not have to deal with integrals involving radiated field in the whole space. It is the main principle that constitutes part of the framework called the Source Concept and this paper summarizes some of the findings in the field. We concentrate only on electric currents flowing in vacuum.

Brillouin [3] was probably the first scientist who proposed to use source spatial currents to evaluate radiated power, see also [4], [5, Sec. 2.3]. An illustration of this procedure is provided later in Sec. 2.1.

Radiation quality factor $Q$ [6], which indicates the bandwidth potential of an antenna, is another important measure. It is well known [6], [7] that the stored energy of an electromagnetic field is infinite in frequency domain (a time-harmonic state) due to radiated energy. Traditionally, radiated energy was subtracted from the total energy and the methods operated with "sphere enclosing the antenna", but without taking into account the exact shape of the radiator, see [8] and references therein. Geyi [9, Sec. 4] and later Vandenbosch [10] and Gustafsson [11] attempted to obtain stored electromagnetic energies directly from currents and their results lead to so-called measurable or observable energies since they are tightly connected to the frequency changes of an antenna's input impedance. Prior to this, observable energies were proposed by Rhodes [12], but he still relied upon using fields in space, not source currents. Section 2.2 illustrates how the measurable energies can be derived in a simple and intuitive way [15], using only spatially localized currents.

It should be noted that the problem of correct definition of stored energy has still not been completely solved, although, currently, it is being intensively studied, see e. g. [13]. In particular, the time-domain approach seems very promising [14].

In Section 3, a well-known Theory of Characteristic Modes [16] is introduced, though expressed in terms of a power functional involving source current density. Such a formulation allows the study of arbitrary current distribution (for instance one may guess for characteristic currents and test their properties) and may enable the study of the optimal composition of modal currents for minimal Q [17].

The last five years have allowed the capabilities of the Source Concept to be recognized and consequently software tools associated with its implementation have begun to appear. To support this effort, the Antenna Toolbox for Matlab (AToM) [18] is currently being developed. The AToM is written entirely in Matlab, so the user can enjoy its semi-open architecture and friendly operation through graphical interface or direct access to low level functions. Main simulation core is based on Method of Moments [4], both for 3D wire and planar structures. Together with modal decomposition (characteristic modes), the source concept, feeding synthesis and powerful optimization, it will present a unique tool for synthesis of antennas.

\section{Source Concept}

Source concept can be introduced as a framework utilizing integral equations involving spatially localized sources of radiation.

It will be shown how to express input impedance, radiated power, measurable energies, quality factor and characteristic eigenvalues solely in terms of electric current 
density. It must be noted that currents can be obtained from a full-wave simulation, by modal decomposition or even specified by analytical approximation. They can be the subject of modal and structural decomposition and optimization which created the possibility of finding optimal current distributions with regard to quality factor $Q$, gain $G, G / Q$ ratio and polarization properties [19].

\subsection{Illustration of the Source Concept}

The following example illustrates the philosophy of the source concept. For the sake of simplicity consider only the linear z-directed current which generates the $\theta$ component in the far-field. It is well known [20] that radiated power can be calculated from far-field power density (the so called "Poynting vector method") as

$$
P_{\mathrm{r}}=\frac{1}{2 Z_{0}} \oiint_{4 \pi}\left|E_{\theta}(r, \theta, \phi)\right|^{2} \mathrm{~d} S
$$

where $Z_{0}$ is the impedance of free space. To eliminate the surface integral involving the radiated field, $\boldsymbol{E}=-\mathrm{j} \omega \boldsymbol{A}$ is inserted into (1)

$$
P_{\mathrm{r}}=\frac{\omega^{2}}{2 Z_{0}} \oiint_{4 \pi}\left|A_{\theta}(r, \theta, \phi)\right|^{2} \mathrm{~d} S
$$

where the vector potential is

$$
A_{\theta}(r, \theta, \phi)=\frac{\mu}{4 \pi} \frac{e^{-\mathrm{j} k r}}{r} \sin \theta \int_{-L / 2}^{L / 2} I\left(z^{\prime}\right) e^{\mathrm{j} k z^{\prime} \cos \theta} \mathrm{d} z^{\prime} .
$$

We now have to evaluate

$$
P_{\mathrm{r}}=\frac{\mu^{2}}{16 \pi^{2} r^{2}} \oiint_{4 \pi} \int_{-L / 2}^{L / 2} \int_{-L / 2}^{L / 2} I(z) I^{*}\left(z^{\prime}\right) \sin ^{2} \theta e^{\mathrm{j} k \cos \theta\left(z-z^{\prime}\right)} \mathrm{d} z \mathrm{~d} z^{\prime} \mathrm{d} S .
$$

There are two integrals over the spatial domain of sources and one surface integral, which can be worked out in closed form

$$
\int_{0}^{\pi} \sin ^{3} \theta e^{\mathrm{j} k R \cos \theta} \mathrm{d} \theta=4\left(\frac{\sin k R}{(k R)^{3}}-\frac{\cos k R}{(k R)^{2}}\right)
$$

where $R=\left|\boldsymbol{z}-\boldsymbol{z}^{\prime}\right|$ has been substituted as a distance between interacting currents.

Hence, the radiated power can now be alternatively written as follows

$$
P_{\mathrm{r}}=30 k^{2} \int_{-L / 2}^{L / 2} \int_{-L / 2}^{L / 2} I(z) I^{*}\left(z^{\prime}\right)\left(\frac{\sin k R}{(k R)^{3}}-\frac{\cos k R}{(k R)^{2}}\right) \mathrm{d} z \mathrm{~d} z^{\prime} .
$$

As a check, constant current $I(z)=I\left(z^{\prime}\right)=I$ is inserted and the kernel is expanded into the Taylor series. The first term integrates to zero and the second gives $P_{\mathrm{r}}=10(\mathrm{kIL})^{2}$, a well known result [20] for an elementary dipole when $L \rightarrow 0$, though obtained in a completely different way.

\subsection{Complex Power and Input Impedance}

Brillouin [3] and Papas [5] have shown that the "Poynting vector method" and the above presented method (which will be revealed in its extended form involving reactive power as the "EMF method" [20]) are equivalent. Physically, the EMF method is based on shrinking the enclosing surface to the boundary of the antenna while allowing to capture the reactive components, too.

The derivation starts with the Complex Poynting Theorem (PT) in frequency domain [6]

$$
P_{\text {in }}=-\frac{1}{2} \int_{V} \boldsymbol{E} \cdot \boldsymbol{J}^{*} \mathrm{~d} V=P_{\mathrm{r}}+\mathrm{j} 2 \omega\left(W_{\mathrm{m}}-W_{\mathrm{e}}\right)=P_{\mathrm{r}}+\mathrm{j} P_{\mathrm{x}}
$$

where $P_{\text {in }}=P_{\mathrm{r}}+\mathrm{j} P_{\mathrm{x}}$ is complex power measured at the antenna ports, $V$ is the volume of the antenna and $W_{\mathrm{m}}$ and $W_{\mathrm{e}}$ are energies of the magnetic and electric fields respectively.

The $\boldsymbol{E} \cdot \boldsymbol{J}^{*}$ part of the above equation for input power may also be illustratively written in terms of dynamic potentials [6] if we substitute $\boldsymbol{E}=-\mathrm{j} \omega \boldsymbol{A}-\nabla \varphi$ into (7):

$$
P_{\text {in }}=\frac{\mathrm{j} \omega}{2} \int_{V}\left(\boldsymbol{A} \cdot \boldsymbol{J}^{*}-\varphi \rho^{*}\right) \mathrm{d} V,
$$

in which $\varphi$ is scalar electric potential and $\rho$ is charge density. The PT thus gives us access to radiated power and difference of magnetic and electric energies, the only quantities which are measurable through the input impedance, compactly expressed as

$$
Z_{\text {in }}=\frac{\mathrm{j} \omega}{\left|I_{0}\right|^{2}} \int_{V}\left(\boldsymbol{A} \cdot \boldsymbol{J}^{*}-\varphi \rho^{*}\right) \mathrm{d} V
$$

where $I_{0}$ is the input current at the antenna terminals.

By inserting the continuity equation $\rho=-\nabla \cdot J / j \omega$, the self-impedance may now be written as double integral involving current density, frequency and antenna geometry:

$$
Z_{\text {in }}=\frac{\mathrm{j} 30}{k\left|I_{0}\right|^{2}} \iint_{V V}[\Psi-\Upsilon] \frac{e^{-\mathrm{j} k R}}{R} \mathrm{~d} V \mathrm{~d} V^{\prime}
$$

where $\Psi=k^{2} \boldsymbol{J}(\boldsymbol{r}) \cdot \boldsymbol{J}^{*}\left(\boldsymbol{r}^{\prime}\right)$ originated from vector potential, $\Upsilon=\nabla \cdot \boldsymbol{J}(\boldsymbol{r}) \nabla^{\prime} \cdot \boldsymbol{J}^{*}\left(\boldsymbol{r}^{\prime}\right)$ originated from scalar potential and the integration is performed over the antenna occupying volume $V$ with $R=\left|\boldsymbol{r}-\boldsymbol{r}^{\prime}\right|$ being the distance between currents $^{1}$

Equation (10) is a departure point for the impedance quality factor [21]. It occurred many times, and in various forms and is known as the "EMF method" or EFIE for evaluating the impedance of an antenna using the prescribed current [20] or in the MoM formulation [22]. The spatial derivative of current can also be transferred to Green's function $\exp (-\mathrm{j} k R) / R$, though the resulting kernel

1 Modification of (10) for evaluating the mutual impedance is straightforward [20]. 


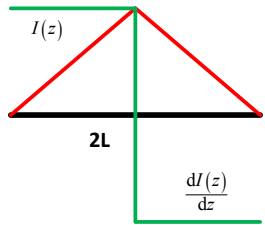

Fig. 1. Distribution of current and charge along a short dipole.

has strong singularities [5], see also (6) where such a differentiated kernel appeared naturally.

Inspecting equation (10) reveals that the contribution to the input impedance (or input complex power) is both magnetic (from currents, $\Psi$ term) and electric (from charges, $\Upsilon$ term).

The following illustrative example considers the evaluation of the radiation resistance of a short dipole with a triangular current, see Fig. 1 .

From (10) we have

$$
R_{\mathrm{r}}=\frac{30}{k\left|I_{0}\right|^{2}} \iint_{V V}[\Psi-\Upsilon] \frac{\sin k R}{R} \mathrm{~d} z \mathrm{~d} z^{\prime}=R_{\mathrm{r}}^{\Psi}-R_{\mathrm{r}}^{\Upsilon}
$$

where $R_{\mathrm{r}}^{\Psi}$ and $R_{\mathrm{r}}^{\mathrm{Y}}$ are contributions from a current and charge respectively. By using two terms of the Taylor series for the kernel $\sin (k R) / R$ we obtain $R_{\mathrm{r}}=R_{\mathrm{r}}^{\Psi}-R_{\mathrm{r}}^{\mathrm{Y}}=30(k L)^{2}-10(k L)^{2}=20(k L)$, another wellknown result, but this is obtained only from sources. Noted that the closed-form calculation of reactance is much more delicate because the dominant (static) part of the kernel $\cos (k R) / R \cong 1 / R$ contains singularity. However, results for first-order linear sinusoidal currents are known and widely available [20].

\subsection{The Radiation Quality Factor}

The untuned quality factor in the frequency domain is generally defined as [6]

$$
Q=\frac{\omega W_{\text {sto }}}{P_{\text {lost }}}=\frac{\omega\left(W_{\mathrm{m}}+W_{\mathrm{e}}\right)}{P_{\mathrm{r}}}
$$

where $W_{\text {sto }}$ is the stored energy and where we assumed that power is lost only by radiation. While radiated power is uniquely defined, the energy of the radiating system in a time-harmonic state is infinite. Therefore, modified finite energies have been proposed by many authors, see e.g. [14]. Unfortunately, deficiencies such as coordinate dependence [21] and negative values [23] exist.

In (12), we may interpret $\omega W_{\text {sto }}$ as a kind of reactive power, hence (12) is in fact a power ratio. But in practice, an antenna designer is usually not primarily interested in the value of stored energy, but in the bandwidth. For this purpose, reasonable definition ${ }^{2}$ of quality factor $Q$, based

${ }^{2}$ Another question arises of whether the quality factor is exactly proportional to the bandwidth. It can be shown [24] that it is, but only for a series/parallel single-resonant circuit or for higher values of $Q$. on the frequency sensitivity of input impedance, have been proposed [12], [25]

$$
Q_{\mathrm{Z}}=\frac{\omega}{2 R_{\text {in }}}\left|\frac{\partial Z_{\text {in }}}{\partial \omega}\right|=\frac{k}{2 R_{\text {in }}}\left|\frac{\partial Z_{\text {in }}}{\partial k}\right|=\left|Q_{\mathrm{R}}+\mathrm{j} Q_{\mathrm{X}}\right|
$$

where $Q_{\mathrm{R}}$ and $Q_{\mathrm{x}}$ represent change of input resistance and reactance, which is dominant, respectively.

Insert $Z_{\text {in }}=2 P_{\text {in }} /\left|I_{0}\right|^{2}$ to obtain

$$
Q_{\mathrm{Z}}=\frac{k}{2 R_{\text {in }}}\left|\frac{\partial}{\partial k} \frac{2 P_{\text {in }}}{\left|I_{0}\right|^{2}}\right|=\frac{P_{\mathrm{A}}}{P_{\mathrm{r}}}
$$

where $P_{\mathrm{A}}=\frac{\partial}{\partial k} \sqrt{P_{\mathrm{r}}^{2}+\mathrm{j} P_{\mathrm{x}}^{2}}$ is the frequency derivative of the apparent input power. The port current $I_{0}$ is assumed to be constant.

Indeed the quality factor $Q_{\mathrm{z}}$ can be evaluated by differentiation of the input impedance obtained from the electromagnetic field simulator. However, we can use (10) to great advantage and perform the derivation in (13) analytically ${ }^{3}$. It is then possible to insert a modal or even arbitrary current density and examine how much a given part of the antenna affects the overall $Q$.

The result is composed of three complex terms (which we loosely call measurable or observable energies [13]) of different nature

$$
\frac{\partial Z_{\text {in }}}{\partial k}\left|I_{0}\right|^{2}=\hat{W}_{\mathrm{me}}+\hat{W}_{\mathrm{r}}+\hat{W}_{\mathrm{k}}
$$

where

$$
\begin{gathered}
\hat{W}_{\mathrm{me}}=\frac{\mathrm{j} 30}{k^{2}} \iint_{V V}[\Psi+\Upsilon] \frac{e^{-\mathrm{j} k R}}{R} \mathrm{~d} V \mathrm{~d} V^{\prime}, \\
\hat{W}_{\mathrm{r}}=\frac{30}{k} \iint_{V V^{\prime}}[\Psi-\Upsilon] e^{-\mathrm{j} k R} \mathrm{~d} V \mathrm{~d} V^{\prime}, \\
\hat{W}_{\mathrm{k}}=\frac{\mathrm{j} 30}{k} \iint_{V V} \frac{\partial}{\partial k}[\Psi-\Upsilon] \frac{e^{-\mathrm{j} k R}}{R} \mathrm{~d} V \mathrm{~d} V^{\prime} .
\end{gathered}
$$

The structure of the first term $\hat{W}_{\text {me }}$ is similar to the $Z_{\text {in, }}$, but includes the sum of parts $\Psi$ and $Y$. This strongly resembles the Foster theorem [21] where the reactance derivation produces a sum of energies. It should be stressed that the Foster theorem is valid only for a lossless reactance network, which is not the case of an radiating system. This term is nonzero, even for non-radiating system and it comprises a major contribution.

The second term $\hat{W}_{\mathrm{r}}$ arises from the frequency change of Green's function and is associated with radiation, see discussion in [15]. It is zero for a non-radiating system. The third term $\hat{W}_{\mathrm{k}}$ accounts for the change of current density with frequency [13] and is, again, zero for a non-radiating system.

${ }^{3}$ This derivation will be performed in $k$ instead of $\omega$ in order to keep the easy notation of (10). 


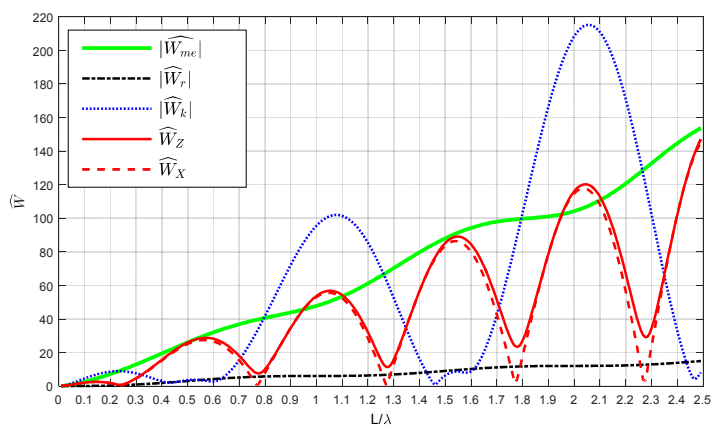

Fig. 2. The module of measurable energies for a dipole of length $L$. In general, the radiation term $\left|\hat{W}_{\mathrm{r}}\right|$ is small. The $\left|\hat{W}_{\mathrm{k}}\right|$ term is small in resonance, where the current distribution is stable and peaks in antiresonances. In resonance, the dominant contribution comes from $\left|\hat{W}_{\text {me }}\right|$.

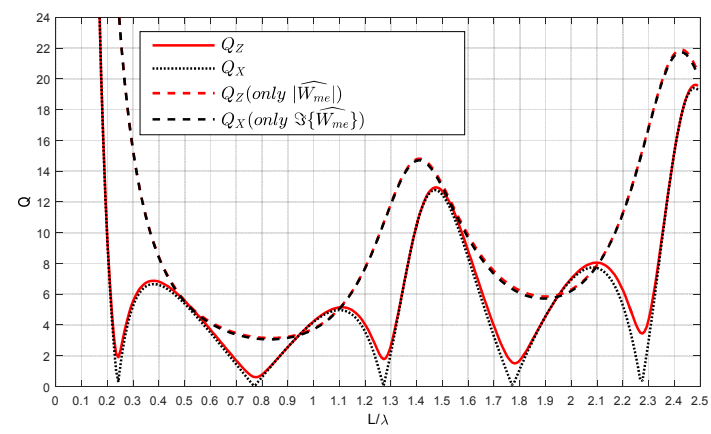

Fig. 3. Untuned quality factors for a thin-wire dipole.

We define the measurable energies, available from the antenna terminals and consider input impedance, or only input reactance, as

$$
\begin{gathered}
\hat{W}_{\mathrm{Z}}=\left|\hat{W}_{\mathrm{me}}+\hat{W}_{\mathrm{r}}+\hat{W}_{\mathrm{k}}\right|, \\
\hat{W}_{\mathrm{x}}=\mathfrak{I}\left\{\hat{W}_{\mathrm{me}}+\hat{W}_{\mathrm{r}}+\hat{W}_{\mathrm{k}}\right\} .
\end{gathered}
$$

The behavior of $\left|\hat{W}_{\text {me }}\right|,\left|\hat{W}_{\mathrm{r}}\right|,\left|\hat{W}_{\mathrm{k}}\right|, \hat{W}_{\mathrm{z}}$, and $\hat{W}_{\mathrm{x}}$, is illustrated in the case of a thin-wire dipole with a prescribed sinusoidal current, see Fig. 2. For easy plotting we show only modules of terms (16)-(18).

Various quality factors were calculated from the above energies. They all agree very well in resonance, as seen in Fig. 3.

Explicitly, the simplest version of the measurable quality factor considers only an imaginary part of the $\hat{W}_{\text {me }}$ term and reads

$$
Q_{\mathrm{me}}=\frac{\iint_{V V^{\prime}}[\Psi+\Upsilon] \frac{\cos k R}{R} \mathrm{~d} V \mathrm{~d} V^{\prime}}{2 \iint_{V V^{\prime}}[\Psi-\Upsilon] \frac{\sin k R}{R} \mathrm{~d} V \mathrm{~d} V^{\prime}} .
$$

weighted eigenvalue equation involving real and imaginary parts of the impedance operator (10) [16]. Alternatively, they can also be expressed as a basis which minimizes the following source concept power functional:

$$
\mathcal{F}\left(\boldsymbol{J}_{n}\right)=\frac{P_{\mathrm{x}}}{P_{\mathrm{r}}}=\frac{\iint_{V V^{\prime}}[\Psi-\Upsilon] \frac{\cos k R}{R} \mathrm{~d} V \mathrm{~d} V^{\prime}}{\iint_{V V^{\prime}}[\Psi-\Upsilon] \frac{\sin k R}{R} \mathrm{~d} V \mathrm{~d} V^{\prime}}=\kappa_{n}
$$

where $\kappa_{n}$ is the Rayleigh quotient, which is equal to characteristic number $\lambda_{n}$ when a true characteristic current $\boldsymbol{J}_{n}$ enters into (22). The functional is minimized by characteristic currents that simultaneously maximizes radiated power and minimizes reactive power, representing external resonances of the radiator. For very special cases, a stationary solution of (22) can be obtained even in closed form. It is clear that we may assign antenna parameters (energies, powers, losses, directivities, near and far fields) directly to the modes and gain extra physical insight into the antenna behavior. In [26] we derived the so-called coupling matrix $\beta_{m n}$ which connects the modes with the real world and is represented by modal excitation coefficients $V_{m}$ [27]

$$
\beta_{m n}=\frac{V_{m} V_{n}\left(1+\lambda_{m} \lambda_{n}\right)}{\left(1+\lambda_{m}^{2}\right)\left(1+\lambda_{n}^{2}\right)} .
$$

This matrix is a good subject for efficient optimization since it is possible to move the feeding along a given structure, control the amount of modal parameters and quickly evaluate total behavior, which became just simple matrix operation. The radiation quality factor $Q$, radiation efficiency and directivity can, then, be compactly expressed using modal quantities as [26], [28]

$$
\begin{gathered}
Q=2 \omega \frac{\sum_{m, n} \beta_{m n} \hat{W}_{m n}^{\mathrm{e}}+\sum_{m, n} \beta_{m n} \hat{W}_{m n}^{\mathrm{m}}}{\sum_{m, n} \beta_{m n} P_{m n}^{\mathrm{r}}}, \\
\eta=\frac{\sum_{m} \beta_{m m}}{\sum_{m} \beta_{m m}+\sum_{m, n} \beta_{m n} P_{m n}^{\mathrm{L}}}, \\
D=15 k^{2} \frac{\sum_{m, n} \beta_{m n} U_{m n}}{\sum_{m} \beta_{m m}}
\end{gathered}
$$

where $\hat{W}_{m n}^{\mathrm{e}}$ and $\hat{W}_{m n}^{\mathrm{m}}$ are modified modal energies, $P_{m n}^{\mathrm{r}}$ is modal radiated power, $P_{m n}^{\mathrm{L}}$ is modal lost power and

$$
U_{m n}=\iint_{V V} \boldsymbol{J}_{m}(\boldsymbol{r}) \cdot \boldsymbol{J}_{n}^{*}\left(\boldsymbol{r}^{\prime}\right) e^{\mathrm{j} k\left(R \cdot r_{0}\right)} \mathrm{d} V \mathrm{~d} V
$$

in (26) is related to the modal radiation intensity.

\section{Conclusions}

An overview of the source concept definition has been presented. The source concept, as proposed, considers sponding eigenvalues $\lambda_{n}$ are usually defined through the 
all parameters expressed solely as a function of the source quantities, namely the electric and magnetic currents. This approach offers many appealing properties, notably fast and uncomplicated evaluations via bilinear integral forms, the possibility to perform structural and modal decomposition, linear, quadratic and heuristic optimization and other advanced techniques of a current's modification and analysis. Recent applications of various antenna parameters expressed by source current have been mentioned in conjunction with potential applications.

\section{Acknowledgments}

This work has been supported by project no. TA 04010457 of the Technology Agency of the Czech Republic and by project SGS16/226/OHK3/3T/13.

\section{References}

[1] MIKKI, S. M., ANTAR, Y. M. M. On cross correlation in antenna arrays with applications to spatial diversity and MIMO systems. IEEE Transactions on Antennas and Propagation, 2015, vol. 63, no. 14, p. 1798-1810. DOI: 10.1109/TAP.2015.2398113

[2] CISMASU, M., GUSTAFSSON, M. Antenna bandwidth optimization with single frequency simulation. IEEE Transactions on Antennas and Propagation, 2013, vol. 62, no. 3, p. 1304-1311. DOI: $10.1109 /$ TAP.2013.2295426

[3] BRILLOUIN, L. Origin of the radiation resistance. Radioelectricité, April 1922, vol. 3, p. 147-152.

[4] HARRINGTON, R. F. Matrix methods for field problems. Proceedings of the IEEE, 1967, vol. 55, no. 2, p. 136-149. DOI: 10.1109/PROC.1967.5433

[5] PAPAS, Ch. Theory of Electromagnetic Wave Propagation. Dover, 1965. ISBN: 0486656780

[6] VOLAKIS, J., CHEN, Ch., FUJiMOTO, K. Small Antennas: Miniaturization Techniques and Applications. McGraw-Hill, 2010. ISBN: 978-0071625531

[7] GUSTAFSSON, M., SOHL, Ch., KRISTENSSON, G. Physical limitations on antennas of arbitrary shape. Proceedings of the Royal Society A, 2007, vol. 463, no. 2086. DOI: 10.1098/rspa.2007.1893

[8] THAL, H. Polarization, gain, and Q for small antennas. IEEE Transactions on Antennas and Propagation, 2011, vol. 59, no. 12, p. 4844-4848. DOI: 10.1109/TAP.2011.2165508

[9] GEYI, W. Handset antenna design: Practice and theory. Progress in Electromagnetics Research PIER80, 2008, p. 123-160. [Online] Cited 2016-02-26. Available at: http://jpier.org/PIER/pier80/07.07111302.pdf

[10] VANDENBOSCH, G. A. E. Reactive energies, impedance and Q factor of radiating structures. IEEE Transactions on Antennas and Propagation, 2010, vol. 58, no. 4, p. 1112-1127. DOI: 10.1109/TAP.2010.2041166

[11] GUSTAFSSON, M., JONSSON, B. L.G. Antenna Q and stored energy expressed in the fields, currents and input impedance. IEEE Transactions on Antennas and Propagation, 2015, vol. 63, no. 1, p. 240-249. DOI: 10.1109/TAP.2014.2368111

[12] RHODES, D. Observable stored energies of electromagnetic systems. Journal of the Franklin Institute, 1976, vol. 302, no. 3, p. $225-237$.
[13] CAPEK, M., JELINEK, L., HAZDRA, P., EICHLER, J. The measurable $\mathrm{Q}$ factor and observable energies of radiating structures. IEEE Transactions on Antennas and Propagation, 2013, vol. 62, no. 1, p. 311-318. DOI: 10.1109/TAP.2013.2287519

[14] CAPEK, M., JELINEK, L., VANDENBOSCH, G. A. E. Stored Electromagnetic Energy and Quality Factor of Radiating Structures. [Online] Cited 2016-02-26. Available at: http://arxiv.org/pdf/1403.0572.pdf

[15] HAZDRA, P., CAPEK, M., EICHLER, J. Comments to "Reactive Energies, Impedance, and Q Factor of Radiating Structures" by G. Vandenbosch. IEEE Transactions on Antennas and Propagation, 2013, vol. 61, no. 12, p. 6266-6267. DOI: 10.1109/TAP.2013.2281566

[16] CHEN, Y. FANG, Ch. Characteristic Modes: Theory and Applications in Antenna Engineering. Wiley, 2015. ISBN: 978-1119-03842-9

[17] CAPEK, M., JELINEK, L. Optimal Composition of Modal Currents For Minimal Quality Factor Q. [Online] Cited 2016-02-26. Available at: http://arxiv.org/pdf/1602.04808.pdf

[18] The Antenna Toolbox in Matlab. [Online] Cited 2016-02-26. Available at: http://antennatoolbox.com/

[19] CAPEK, M., JELINEK, L. Optimal Currents on Arbitrarily Shaped Surfaces. [Online] Cited 2016-02-26. Available at: http://arxiv.org/pdf/1602.05520.pdf

[20] JORDAN, E.C., BALMAIN, K. G. Electromagnetic Waves and Radiating Systems. Prentice-Hall, 1968. ISBN: 9780132499958

[21] YAGHJIAN, A. D., BEST, S. R. Impedance, bandwidth, and Q of antennas. IEEE Transactions on Antennas and Propagation, 2005, vol. 53, no. 4, p. 1298-1324. DOI: 10.1109/TAP.2005.844443

[22] HARRINGTON, R. F. Field Computation by Moment Methods. Wiley, 1993. ISBN: 9780780310148

[23] GUSTAFSSON, M., CISMASU, M., JONSSON, B. L. G. Physical bounds and optimal currents on antennas. IEEE Transactions on Antennas and Propagation, 2012, vol. 60, no. 6, p. 2672-2681. DOI: 10.1109/TAP.2012.2194658

[24] CAPEK, M., JELINEK, J., HAZDRA, P. On the functional relation between quality factor and fractional bandwidth. IEEE Transactions on Antennas and Propagation, 2015, vol. 63, no. 6, p. 2787-2790. DOI: 10.1109/TAP.2015.2414472

[25] KAJFEZ, D., WHELESS, W. P. Invariant definitions of the unloaded Q factor. IEEE Transactions on Microwave Theory and Techniques, 1986, vol. 34 , no. 7 , p. 840-841. DOI: 10.1109/TMTT.1986.1133452

[26] CAPEK, M., HAZDRA, P., EICHLER, J. A Method for the evaluation of radiation $\mathrm{Q}$ based on modal approach. IEEE Transactions on Antennas and Propagation, 2012, vol. 60, no. 10, p. 4556-4567. DOI: 10.1109/TAP.2012.2207329

[27] HARRINGTON, R., MAUTZ, J. Theory of characteristic modes for conducting bodies. IEEE Transactions on Antennas and Propagation, 1971, vol. 19, no. 5, p. 622-628. ISSN: 0018-926X, DOI: 10.1109/TAP.1971.1139999

[28] CAPEK, M., EICHLER, J., HAZDRA, P. Evaluating radiation efficiency from characteristic currents. IET Microwaves, Antennas and Propagation, 2015, vol. 9, no. 1, p. 10-15. DOI: 10.1049/ietmap.2013.0473

\section{About the Authors ...}

Pavel HAZDRA received his M.Sc. and Ph.D. from the Czech Technical University (CTU) in Prague in 2003 and 2009 respectively and was appointed an associate professor in 2012. His research interests include antenna and EM 
theory, computational electromagnetics and special primefeed antenna structures.

Miloslav CAPEK received his M.Sc. and Ph.D. from the CTU in Prague in 2009 and 2014 respectively. He leads the development of the AToM (Antenna Toolbox for Matlab) package. His research interests are in the area of electromagnetic theory, electrically small antennas, numerical techniques, fractal geometry and optimization.
Michal MASEK received his M.Sc. from the Czech Technical University in Prague in 2015. He is working towards his $\mathrm{PhD}$ focused on behavior of small antennas close to large objects.

Tomas LONSKY received his M.Sc. from the Czech Technical University in Prague in 2015. He is working towards his $\mathrm{PhD}$ focused on closely-spaced antenna arrays. 